\title{
Latest development on the simulation of rolling contact fatigue crack growth in rails
}

\author{
L. Zhang, S. Mellings, J. Baynham \& R. Adey \\ $C M B E A S Y$ Ltd, UK
}

\begin{abstract}
The two main causes of railway replacement are wear and rolling contact fatigue. Rolling contact fatigue has been a critical problem on UK railways. This paper describes recent developments in the modelling of cracks in rails, which incorporates in the model the contact between the crack faces during calculation of the Stress Intensity Factors (SIFs). This data is then combined with the SIFs caused by contact loading to provide a more realistic simulation of the crack growth. The way the methodology can be applied is described, for example using point loads to represent non-conforming contact to obtain the interaction between the wheel and the rail. The process of the wheel rolling over the crack can be modelled by moving the so called 'contact patch' along the rail. Various conditions can be applied to the crack, including frictionless contact between opposing surfaces, or frictional contact. In addition, pressure can be applied to the crack surfaces to take into account the water trapped inside the crack as the wheel rolls over. An example is given at the end of the paper to illustrate the advantages of the improved model.
\end{abstract}

Keywords: rails, rolling contact fatigue, contact, crack growth, boundary element method (BEM).

\section{Introduction}

The rolling contact fatigue has been a major issue for the British rail industry after the fatal derailment at Hatfield in 2000. On inspections across the national railway system, it was discovered that the rolling contact fatigue was more widespread and serious than previously thought. As a result, the track maintenance practices were modified, urgent rail replacement was undertaken and speed restrictions were imposed $[1,2]$. 
The aim of this paper is to provide a better understanding of the contact rolling fatigue and resulting crack growth by simulating crack growth in a fullscale rail. A 3-D dual boundary element model has been created using BEASY fracture software. For the dual boundary element method the general displacement integral equation and traction integral equation are given by:

$$
\begin{aligned}
& c_{i j}\left(x^{\prime}\right) u_{j}\left(x^{\prime}\right)+\int_{\Gamma} T_{i j}\left(x^{\prime}, x\right) u_{j}(x) d T(x)=\int_{\Gamma} U_{i j}\left(x^{\prime}, x\right) t_{j}(x) d T(x) \\
& \frac{1}{2} t_{j}\left(x^{\prime}\right)+n_{i}\left(x^{\prime}\right) \int_{\Gamma} S_{k i j}\left(x^{\prime}, x\right) u_{k}(x) d T(x) \\
& =n_{i}\left(x^{\prime}\right) \int_{\Gamma} D_{k i j}\left(x^{\prime}, x\right) t_{k}(x) d T(x)
\end{aligned}
$$

where $T_{i j}\left(x^{\prime}, x\right), U_{i j}\left(x^{\prime}, x\right)$ represent, respectively, the traction and displacement fundamental solutions of Kelvin. $u(x)$ and $t(x)$ denote the displacements and tractions. The DBEM model is composed of discontinuous and continuous elements, which can be seen in fig.1.

The current study is based on the original work carried out by Smith [4]. The original model includes a rail and a circular crack initiated from the surface of the rail. The effects of rail surface and crack surface friction on the crack growth were taken into consideration. The improved model developed by the authors is more efficient and realistic in terms of the modelling of the rail, as there are fewer restrictions on the definition of boundary conditions. Friction and contact conditions between crack surfaces can be readily modelled. The improved model can be applied to study the relationship between the crack orientation and the fatigue growth path, and can also be used to predict the fatigue life of the rail, whereby an optimal inspection and maintenance procedure can be worked out.

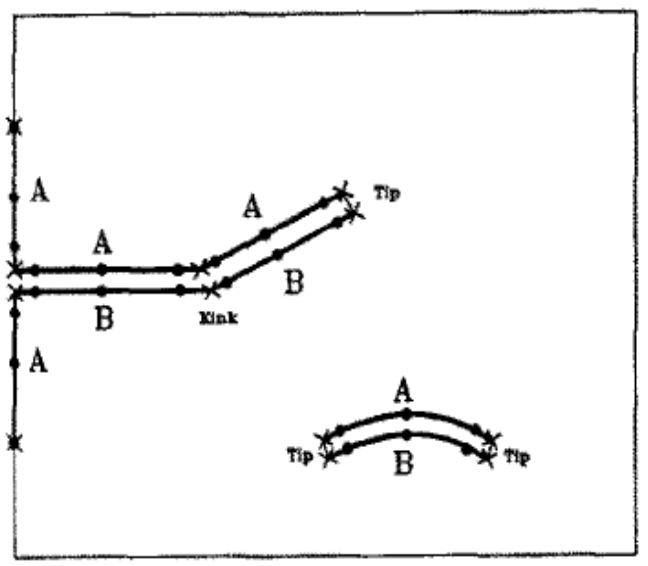

- Element node

$x \cdot$ Element end point

A-Displacement equation

B. Traction equation

Figure 1: Crack modelling with discontinuous and continuous elements [3]. 


\section{Rail model and contact pressure}

The rail chosen for the modelling is a simplification of an EN60E1 (UIC60) rail (fig. 2). This rail has been used in the refurbishment of the West Coast Mainline [2]. The wheel-rail contact is a non-conforming contact where two bodies contact over an area known as the contact patch as shown in fig. 3. A circular contact Hertzian pressure distribution has been chosen to represent the contact loading. The pressure distribution can be obtained through:

$$
P=P_{0} \sqrt{1-\frac{x^{2}}{a^{2}}-\frac{y^{2}}{b^{2}}}
$$

where $a$ and $b$ are half widths of the contact patch. $p_{0}$ is the maximum Hertzian pressure which is given by:

$$
p_{0}=\frac{3}{2} \frac{p}{\pi a b}
$$

where $p$ is the total load on the contact. The contact patch is further divided into very fine grids and the pressure within each grid is integrated giving the total force applied over each grid. The forces over the grids are then applied to a large number of 'load points' and the resulting load can be directly applied on the surface of the elements representing the rail in the model.

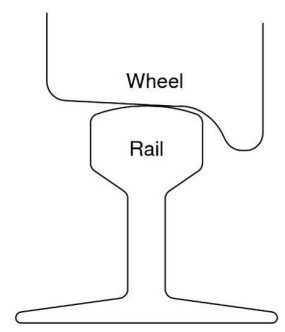

Figure 2: Wheel-rail contact (Smith [4]).

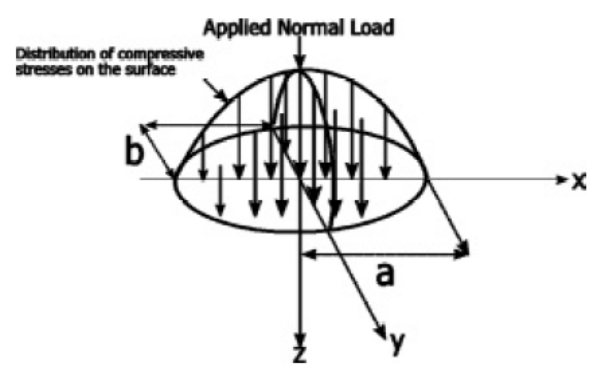

Figure 3: Hertzian elliptical contact pressure [4]. 


\section{Development of an improved rolling contact fatigue (RCF) model}

The RCF in rails can be divided into two categories according to Grassie and Kalousek [5], i.e. cracks initiated at or beneath the rail surface ("surface cracks" and "embedded cracks" respectively). The rail failure due to crack propagation can be seen in fig. 4 . In this study, the surface cracks in the rail are investigated. The main mechanism for crack growth under investigation is dynamic shear loading (fig. 5) arising from the interaction between the wheel and the rail each time the wheel rolls over the crack mouth.

As the wheel rolls over the crack, the stress field surrounding the crack changes significantly. A series of contact patches as seen in fig. 6 are used to capture such changes. The contact patches are evenly distributed along the direction of the motion of the wheel by a certain distance. The load history of the

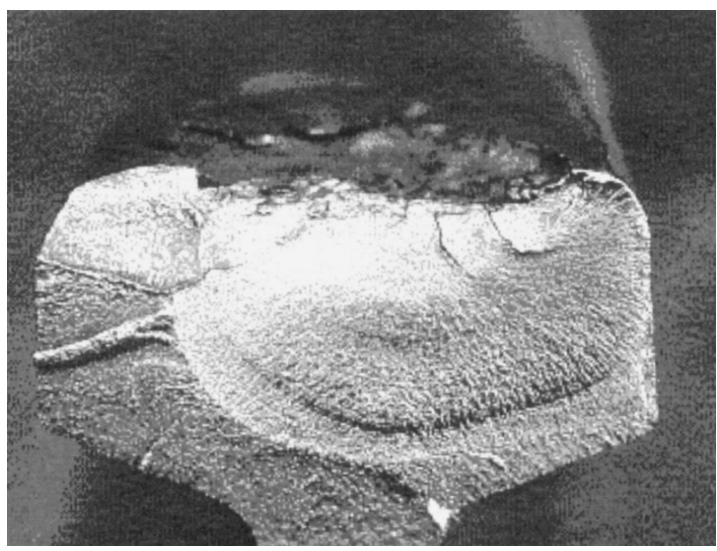

Figure 4: $\quad$ Rail failure as a result of RCF (Cannon et al. [6]).

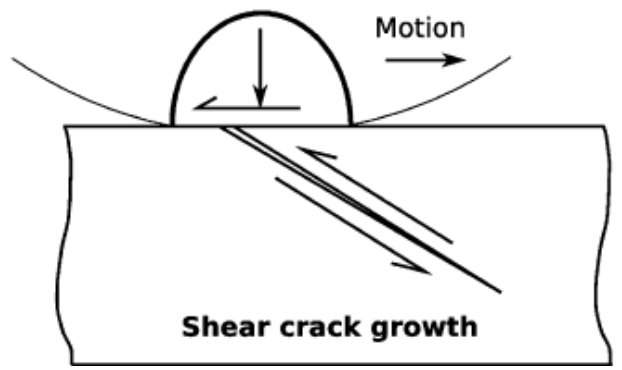

Figure 5: Shear mode crack growth, accelerated by reduction of friction between the crack faces (Fletcher et al. [7]). 


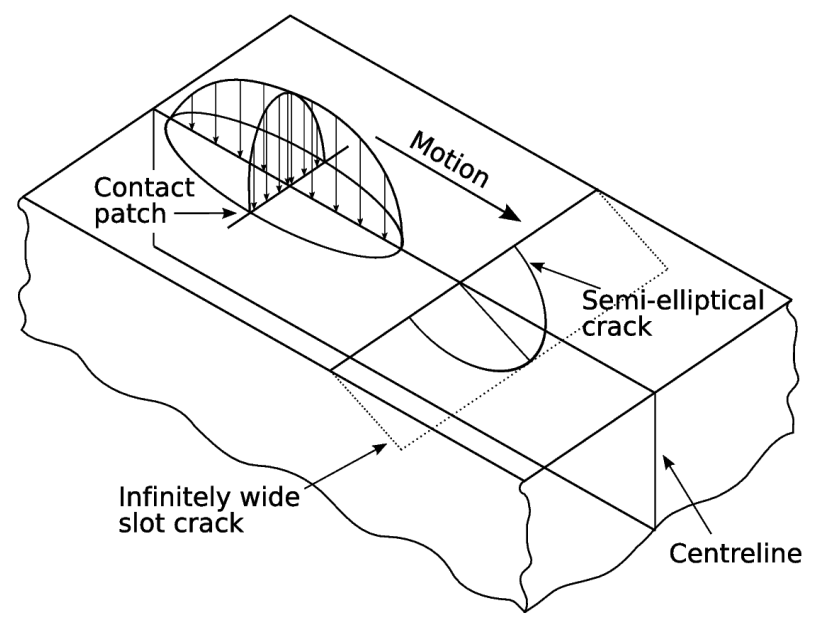

Figure 6: Schematic representation of the RCF model developed by Fletcher et al. [7].

rail can then be obtained by solving each individual problem. The resulting maximum and minimum stress intensity factors can be used to compute the crack growth angle and the fatigue life of the rail.

The original RCF model developed by Fletcher and Kapoor [8] has certain limitations. Firstly, in their model the crack growth trajectory is predefined implying the crack can only grow along the predefined crack surface. Secondly, the rail must be divided into multi-zones to simulate the contact between the crack faces. This entails a significant modelling effort to create the model. Finally, the crack cannot automatically propagate in the rail as the region surrounding the crack needs to be remeshed after each crack growth step. However, the latest development of BEASY fracture software enables the user to solve such a complex practical problem with ease. There is no need to define an artificial surface along which the crack can grow as the contact between crack faces is automatically simulated by the software. The benefit is obvious in that the user interaction with the software during analysis is kept to a minimum while the simulation results such as the stress intensity factor, the crack propagation direction and the contact conditions can be more accurately computed. Fig. 7 shows a comparison between the two models where the new simplified crack modelling can be clearly seen.

Both ends of the rail are fixed in the length direction and the bottom of the rail is fixed in all directions, as its movement is restricted. The crack growth rate follows the Paris' equation and the maximum tangential stress criterion has been utilised to predict the crack growth direction. Following each crack growth step a new crack is inserted to the rail and the region surrounding the crack is automatically remeshed. This process repeats automatically until the crack reaches the required size or the failure criteria are met. 

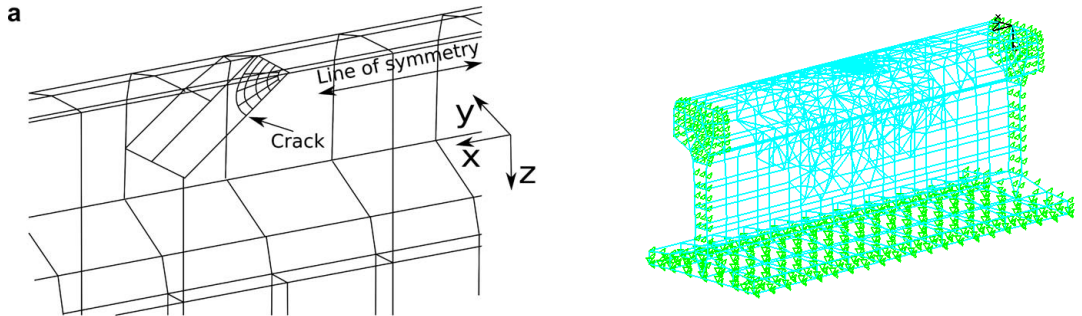

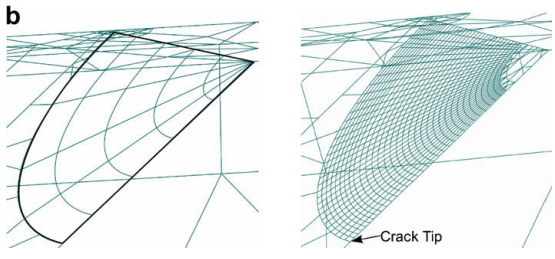

A: Original model developed by Fletcher

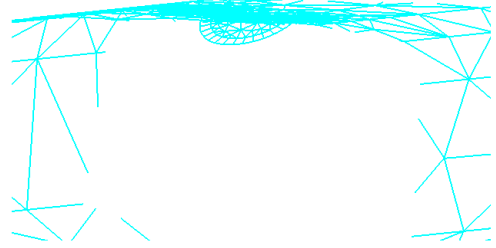

B: Improved 3D BEM model

Figure 7: A) The original symmetric model; and B) the improved 3D BE rail model developed in the current study.

\section{Example}

In this section, a sample problem is solved to illustrate the advantages of the improved approach in solving the rolling contact fatigue problem. The contact pressure, which is similar to the loading used in the original work conducted by Smith [4], has been applied to the surface of the rail.

\subsection{The rail}

The rail chosen for the modelling is a UIC60 rail as shown in fig. 8. A 3-D BE model has been created based on the geometry. The rail section under investigation is $400 \mathrm{~mm}$ long. The dynamic friction coefficient of the rail $\mu_{\mathrm{d}}=0.1$. The young's modulus and the passion ratio for the rail are $210 \mathrm{GPa}$ and 0.3 respectively.

\subsection{The crack}

A thumbnail crack has been inserted to the rail as seen in fig. 9. Such a configuration means a small crack has been initiated at the surface of the rail as a result of a combination of high normal and tangential stresses between the wheel and rail. It is expected that the stresses surrounding the crack will change considerably as the wheels roll over the region near the crack, thus causing the crack to propagate. The initial length and the depth of the crack are $20 \mathrm{~m}$ and $10 \mathrm{~mm}$ respectively. The angle between the rail surface and the crack faces is 30 degrees. The static friction coefficient for the crack surfaces is $\mu_{\mathrm{s}}=0.3$. 


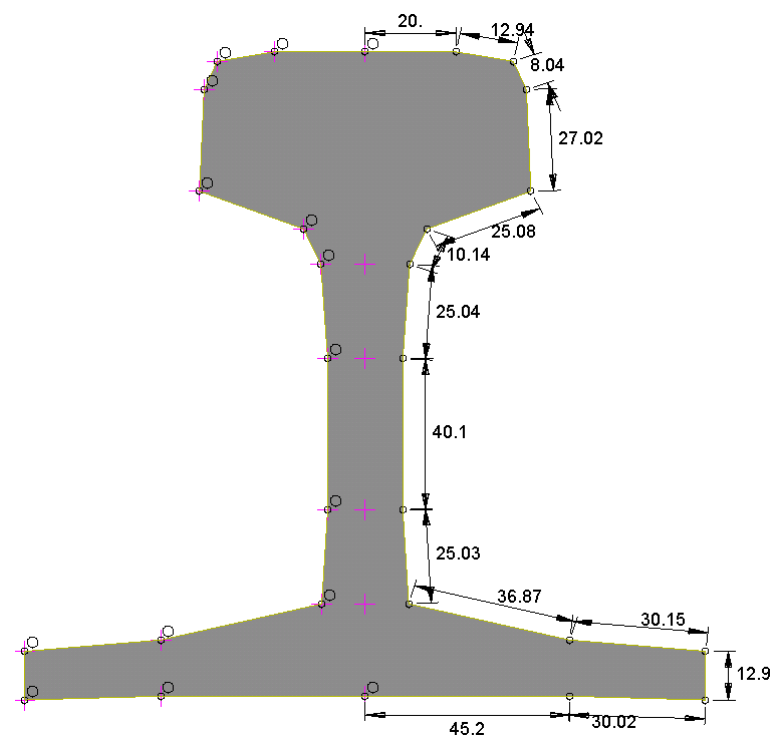

Figure 8: $\quad$ Simplified UIC 60 rail section (unit: $\mathrm{mm}$ ).

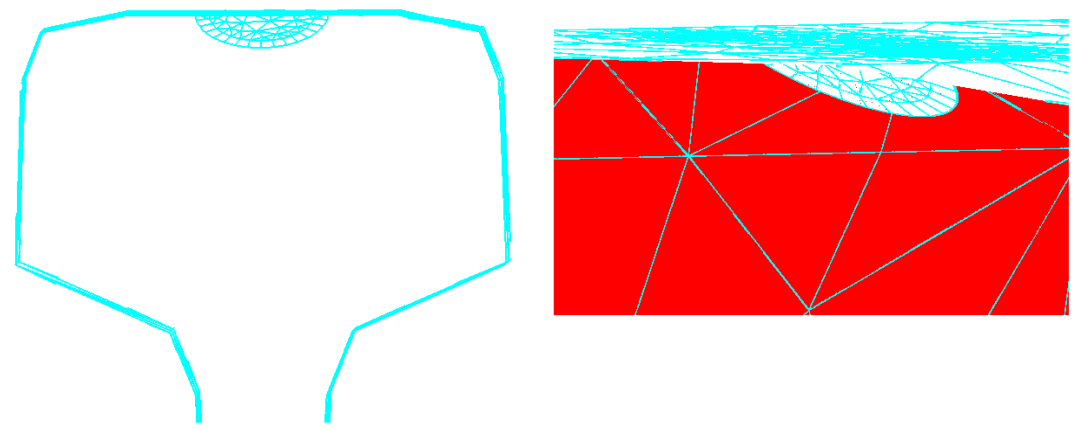

Figure 9: A thumbnail surface crack inserted into the rail model.

\subsection{Boundary conditions and loading}

The boundary conditions are as same as those described in section 3 . The displacement for both ends of the rail in the length direction is restrained and the base of the rail is fixed. The maximum contact pressure is $1750 \mathrm{MPa}$. The contact profile is defined as such that parameters $a$ and $b$ in equation 2.1 are both set equal to $9.5 \mathrm{~mm}$. Twelve load patches have been used to simulate the wheel rolling over the rail. The first path is position $25 \mathrm{~mm}$ to the left of the crack i.e. before the wheel rolls over the crack and the last contact patch is positioned $30 \mathrm{~mm}$ to the right of the rail. One complete load cycle consists of the twelve load cases. The load spectrum file, which is used by the BEASY solver to perform fatigue calculation, follows the format as follows: 

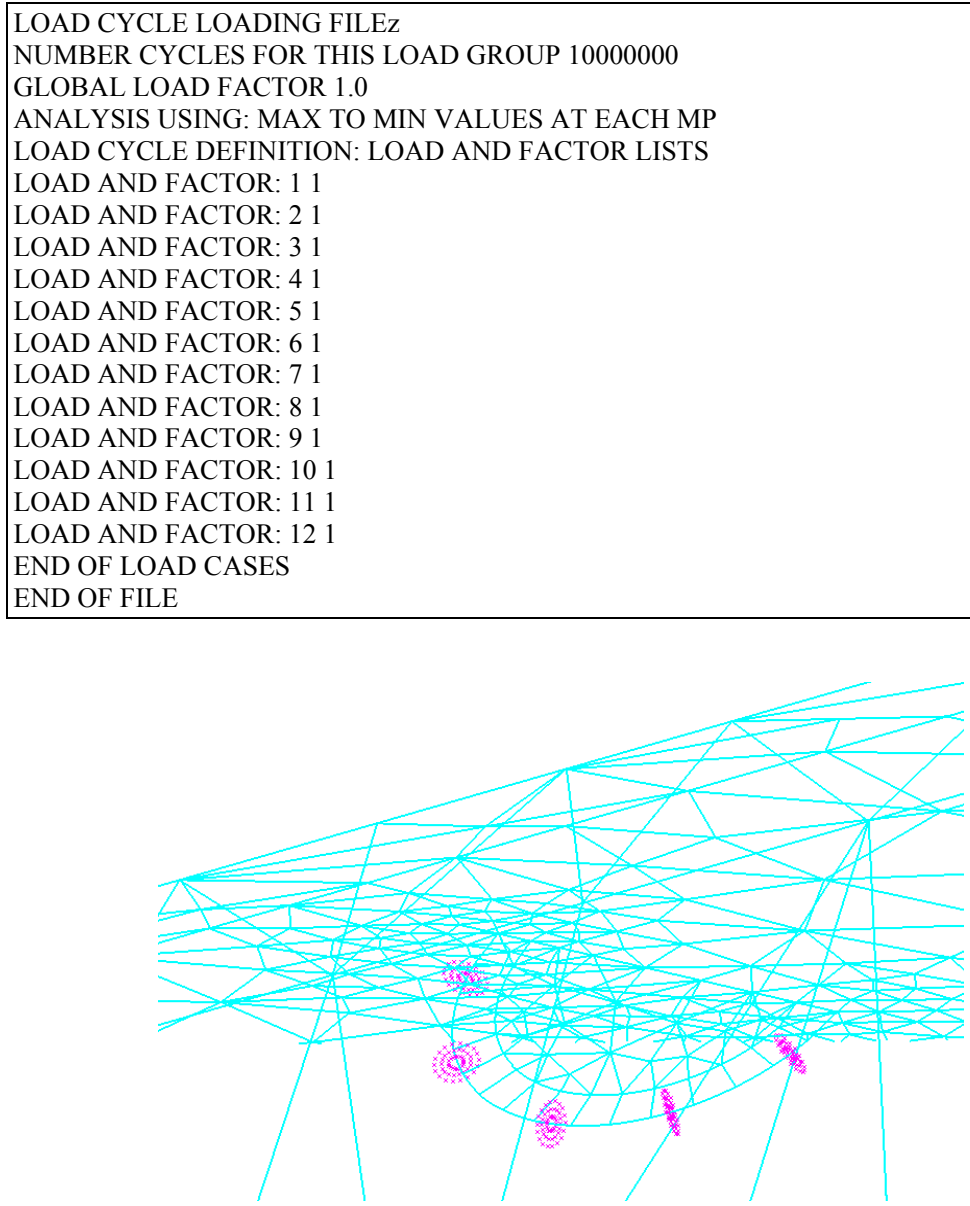

Figure 10: Internal points used to evaluate the J-integral.

The stress intensity factors (Mode I, II, III) along the crack front are computed using a J Integral calculation and used to compute the full mixed mode 3D growth of the crack. Fig 10 shows the model of the crack and some of the locations of the points used to evaluate the J Integral.

\subsection{Results}

\subsubsection{Stress intensity factors along the crack front for crack growth step 0 (i.e. the thumbnail crack of the original depth and length)}

Fig. 11 shows the evolution of the stress intensity factors at the crack tip for crack step 0 as the wheel rolls over the rail. Mode III stress intensity factor is nearly zero as there is no tearing at the crack tip. However, mode I and mode II stress intensity factors vary considerably as the crack surfaces slide against each other. 
The SIF values have been plotted against the relative positions of the mesh points along the crack front.

\subsubsection{Crack growth in the rail}

Fig. 15 shows the crack surfaces after five crack growth increments. It can be seen that the crack growth direction changes dramatically after the first growth step largely due to the sliding of the crack surfaces. The crack then continues to

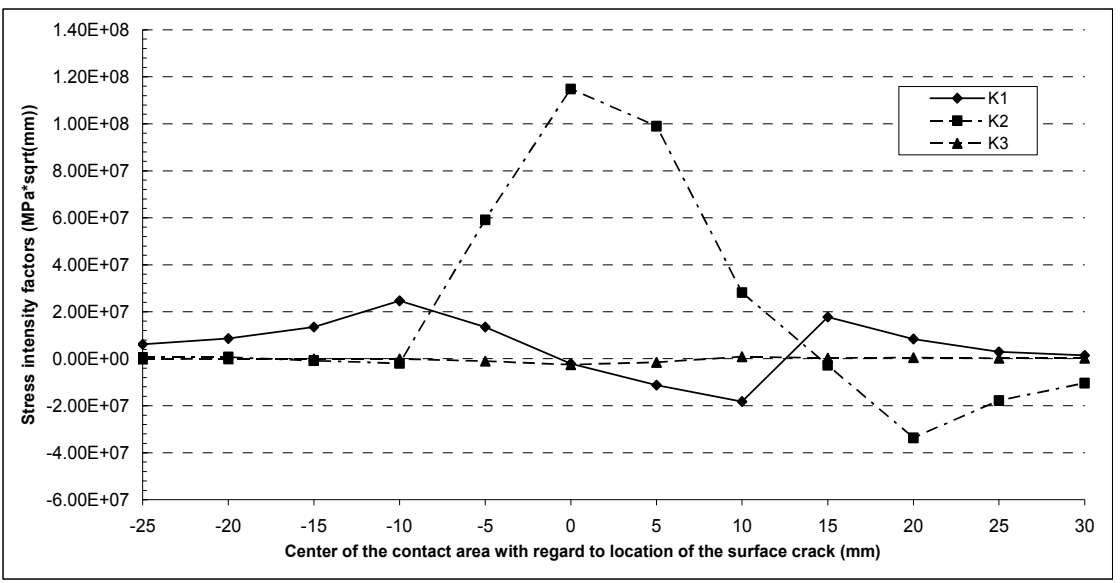

Figure 11: The variation of the SIFs at the crack tip; crack growth step 0.

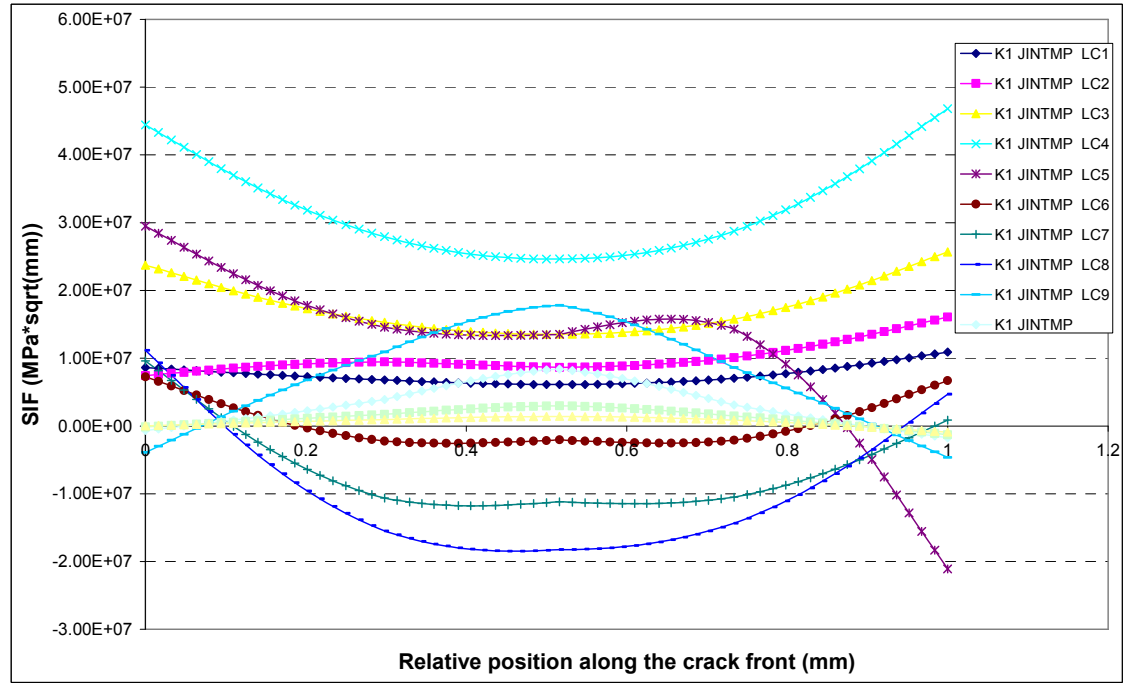

Figure 12: $\quad$ Mode I SIF along the crack front; crack growth step $=0$. 


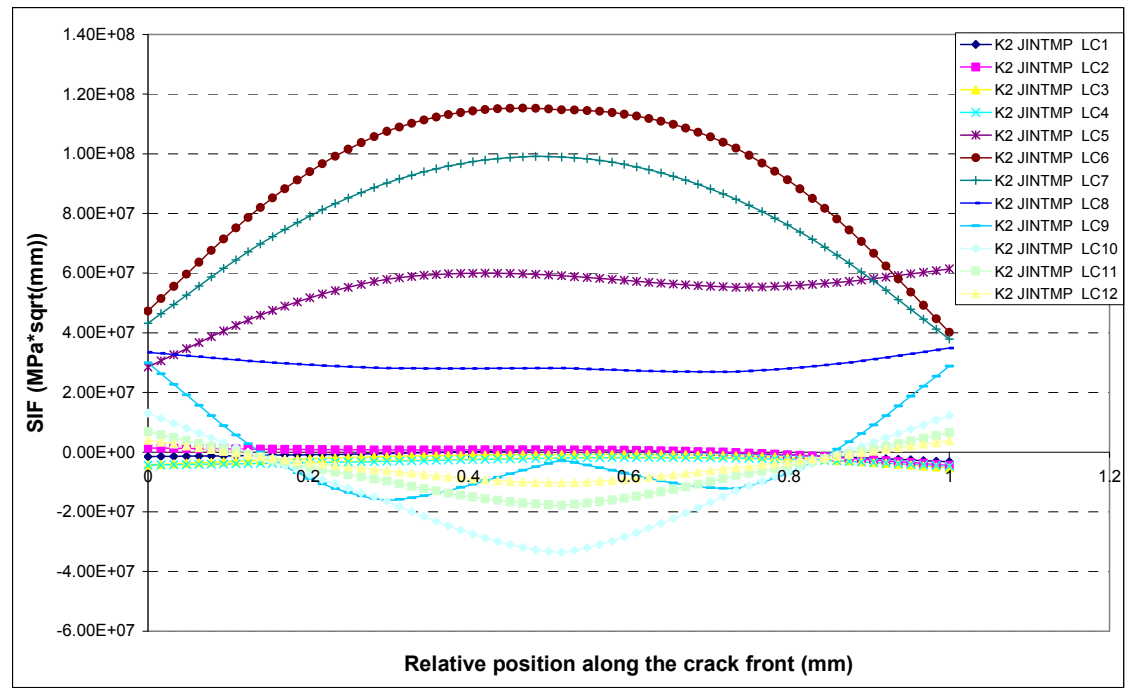

Figure 13: Mode II SIF along the crack front; crack growth step $=0$.

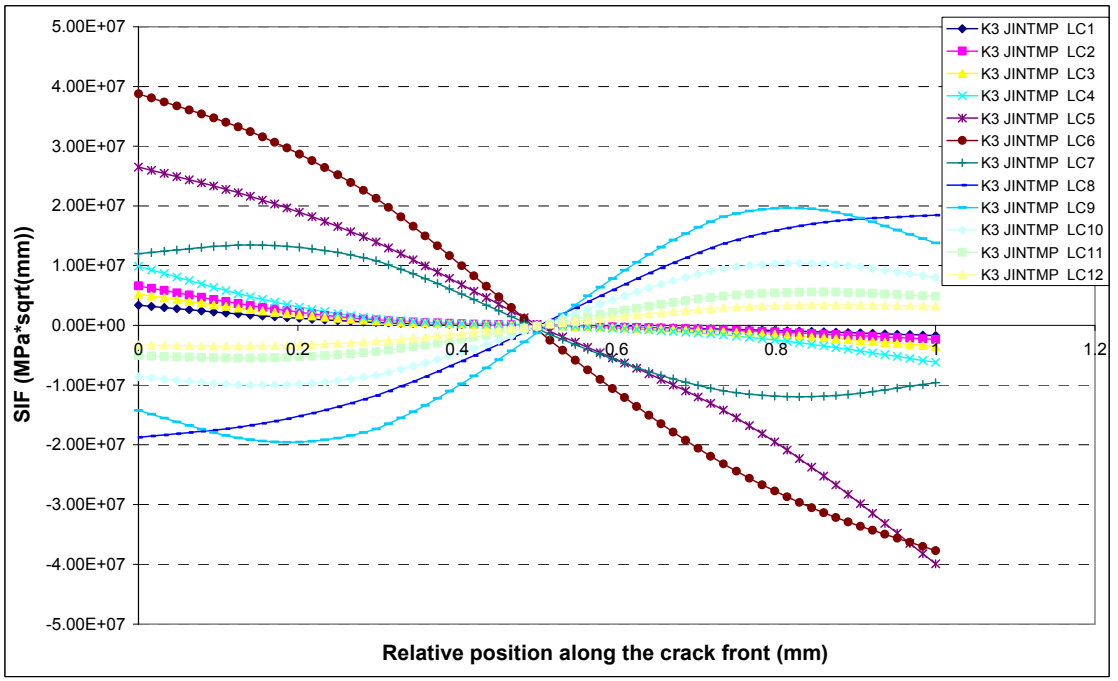

Figure 14: $\quad$ Mode III SIF along the crack front; crack growth step $=0$. 


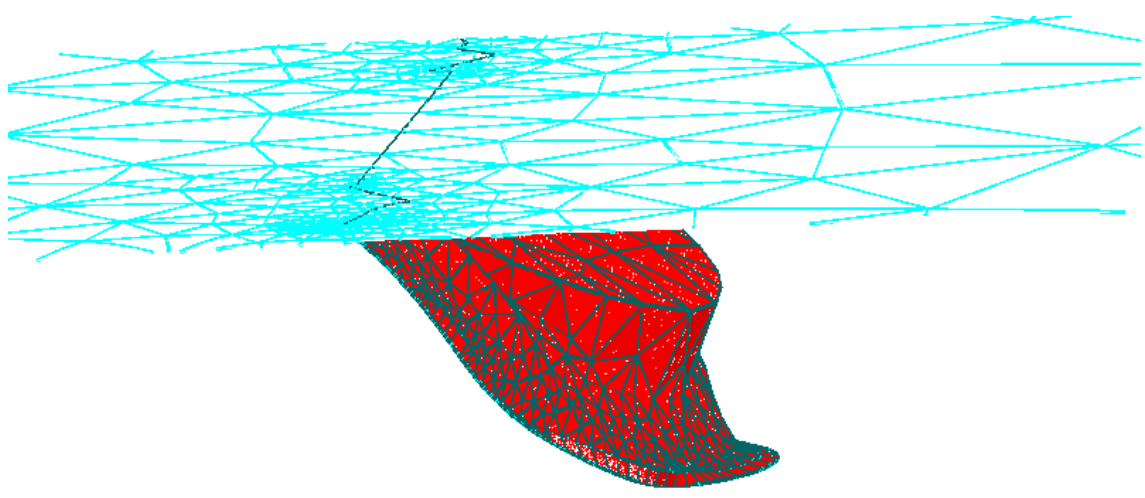

Figure 15: Crack faces after five growth steps.

grow along the crack surfaces. This indicates that the initial crack orientation specified was not exactly correct but the model has automatically reoriented the crack to reflect the actual stress field.

\section{Summary}

This paper introduces an improved version of the rolling contact fatigue rail model. A complete 3-D BE model has been created to simulate the crack growth in a rail which is subjected to rolling contact. The friction between the crack faces and the friction between the wheel and the rail can also be taken into account during simulation. Additional loading can be applied to the crack faces if more detailed analysis is required. The new techniques used not only simplify the modelling process but also improve the accuracy of the results.

\section{Acknowledgement}

The authors would like to thank Dr. David Fletcher from Sheffield University for assistance with details of the rail model used in reference [4].

\section{References}

[1] S. L. Grassie, Rolling contact fatigue on the British railway system: treatment, Wear 258 (2005) 1310-1318.

[2] Network Rail Route Plans - 26 Strategic Routes, March 2005

[3] A. Portela, Dual Boundary Element Analysis of Crack Growth

[4] Lindsey Smith, Rolling Contact Fatigue in Wheel-Rail Contact, $\mathrm{PhD}$ thesis, University of Newcastle Upon Tyne 2007.

[5] S.L. Grassie, J. Kalousek, Rolling contact fatigue of rails: characteristics, causes and treatments, Proceedings of the Sixth International Heavy Haul Railway Conference 1997, pp. 381-404. 
[6] Cannon, D, F, Edel, K, Grassie, S, L, Sawley, K, Rail defects: an overview, Fatigue Fract. Engng. Mater. Struct. 26, (2003) 865-887

[7] Fletcher, DI, P. Hyde and A. Kapoor, Modelling and full-scale trials to investigate fluid pressurisation of rolling contact fatigue cracks, Wear 265 (2008) 1317-1324

[8] Fletcher, DI, and Kapoor, A (2006), Rapid method of stress intensity factor calculation for semi-elliptical surface breaking cracks under threedimensional contact loading, Proceedings of the institution of mechanical engineers, Part F, Journal of Rapid Rail and Rapid Transit, 220 (3): 219-234 